\title{
Feature Fusion Technique for Colour Texture Classification System Based on Gray Level Co-Occurrence Matrix
}

\author{
${ }^{1}$ A. Suresh and ${ }^{2}$ K.L. Shunmuganathan \\ ${ }^{1}$ Department of CSE, Dr. M.G.R Educational and Research Institute University, Chennai, Tamilnadu, India \\ ${ }^{2}$ Department of CSE, R.M.K Engineering College, Chennai, Tamilnadu, India
}

Received 2012-12-03, Revised 2012-12-14; Accepted 2012-12-14

\begin{abstract}
In this study, an efficient feature fusion based technique for the classification of colour texture images in VisTex album is presented. Gray Level Co-occurrence Matrix (GLCM) and its associated texture features contrast, correlation, energy and homogeneity are used in the proposed approach. The proposed GLCM texture features are obtained from the original colour texture as well as the first non singleton dimension of the same image. These features are fused at feature level to classify the colour texture image using nearest neighbor classifier. The results demonstrate that the proposed fusion of difference image GLCM features is much more efficient than the original GLCM features.
\end{abstract}

Keywords: Colour Texture Classification, Gray Level Co-Occurrence Matrix, VisTex Album, Nearest Neighbor Classifier

\section{INTRODUCTION}

Classification of texture images is the most important task in image processing and pattern recognition. Extensive researches have been made for the classification of texture images. Integrative approaches towards color texture classification learned by a supervised framework are introduced in (Bunte et al., 2011). This approach is based on the Generalized Learning Vector Quantization (GLVQ), extended by an adaptive distance measure which is defined in the Fourier domain and 2D Gabor filters. The features learned by GLVQ improve classification accuracy and they generalize much better for evaluation data previously unknown to the system.

A novel statistical approach for color texture modeling and classification based on co-occurrence matrices and discrete finite mixture models is proposed in (Bouguila and Hammoud, 2009). This statistical model assigns relevance weights to discrete co-occurrence features that are considered as random variables. A novel modality invariant texture descriptor which is built by modifying the standard procedure for building local binary pattern is described in (Sadat et al., 2011). The proposed descriptor achieves better accuracy for classifying multimodal images.

An approach which is proposed in study (Ershad, 2011) could analyze the texture based on its grain components and then by making grain components histogram and extracting statistical features from that would classify the textures. Finally, to increase the accuracy of classification, proposed approach is expanded to color images to utilize the ability of approach in analyzing each RGB channels, individually.

The usages of wavelet transform and a neural network ensemble for color texture classification problem is investigated in (Sengur, 2009). The proposed scheme is composed of a wavelet domain feature extractor and ensembles of neural networks classifier. Entropy and energy features are integrated to the wavelet domain feature extractor. Novel approaches to Gabor filtering of color textures are introduced in (Palm and Lehmann, 2002). It is based on the complex chromatic Fourier transform.

A novel color texture classification approach is presented in (Sertel et al., 2008) to computer-assisted Tamilnadu, India 
grading of follicular lymphoma from whole-slide tissue samples. The digitized tissue samples of follicular lymphoma were classified into histological grades under a statistical framework. The proposed method classifies the image either into low or high grades based on the amount of cytological components.

A Wavelet energy correlation signature is introduced and the transformation of these signatures upon linear color space transformations is also derived in (Wouwer et al., 1999). Experiments are conducted on a set of 30 natural colored texture images in which color and gray-level texture classification performances are compared. The study in (Tou et al., 2009) shows that the signal processing methods, such as Gabor filters and wavelets are gaining popularity but old methods such as GLCM are still used but are improved with new calculations or combined with other methods.

Novel results are presented on Neural Network based classification of color textures in a very large heterogeneous database is explained in (Hernandez et al., 2005). Several different Multispectral Random Field models are used to characterize the textures. The classifying features are based on the estimated parameters of these model and functions defined on them. The approach is tested on a database of 73 different color textures classes.

An approach for color texture classification of industrial products is presented in (Akhloufi et al., 2008). An extension of GLCM to color images is proposed. Statistical features are computed from an isotropic colour co-occurrence matrix for classification. The following color spaces are used: RGB, HSL and La*b*. The study on identification and classification of food grains using different color models such as $\mathrm{L}^{*} \mathrm{a} * \mathrm{~b}$, HSV, HSI and $\mathrm{YCbCr}$ by combining color and texture features without performing preprocessing is presented in (Patil et al., 2011). The k-nearest neighbor and minimum distance classifier are used to identify and classify the different types of food grains using local and global features. In this study, a new framework for the classification of colour texture images based on GLCM and nearest neighbor classifier is presented.

\section{MATERIALS AND METHODS}

The proposed system for colour texture classification is built based on GLCM and by applying KNN as classifier. The theoretical background of all the approaches are introduced here.

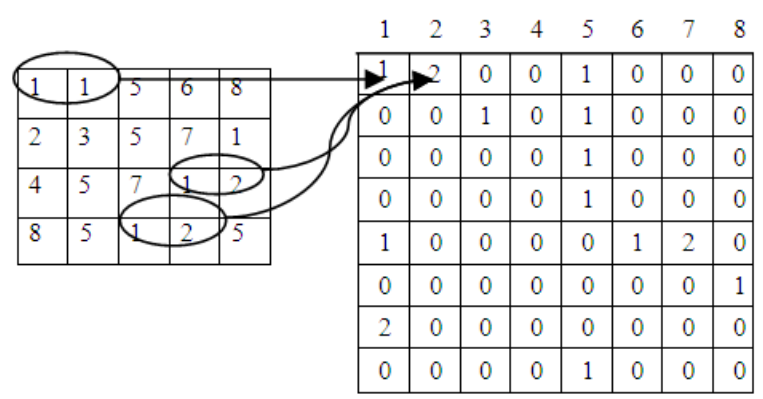

Fig. 1. GLCM for a sample matrix having only 8 gray values

\subsection{Gray Level Co-occurrence Matrix}

The mathematical concept of the basic steps for computing GLCM is illustrated. The first step to extract the statistical features such as contrast, correlation, energy and homogeneity is to compute the GLCM matrix of the input texture image. The GLCM functions characterize the texture of an image by calculating how often pairs of pixel with specific values and in a specified spatial relationship occur in an image, creating a GLCM and then extracting statistical measures from this matrix. The GLCM matrix for an image having 8 gray values is shown in Fig. 1.

The GLCM is normalized so that the sum of its elements is equal to 1 . Each element $(i, j)$ in the normalized GLCM is the joint probability occurrence of pixel pairs with a defined spatial relationship having gray level values $i$ and $j$ in the image. Let us consider $p$ is the normalized GLCM of the input texture image. Contrast is a measure of the intensity contrast between a pixel and its neighbor over the whole image and given by the Equation 1 and the measure of how correlated a pixel is to its neighbor over the whole image is given by the Equation 2:

$$
\text { contrast }=\sum_{i, j}|i-j| p(i, j)^{2}
$$

correlation $=\sum_{i, j} \frac{(i-\mu i)(j-\mu j) p(i, j)}{\sigma_{i} \sigma_{j}}$

The energy is the sum squared element in the normalized GLCM and given by the Equation 3 and the homogeneity in Equation 4 is a value that measures the closeness of the distribution of elements in the GLCM to the GLCM diagonal:

$$
\text { Energy }=\sum_{i, j} p(i, j)^{2}
$$




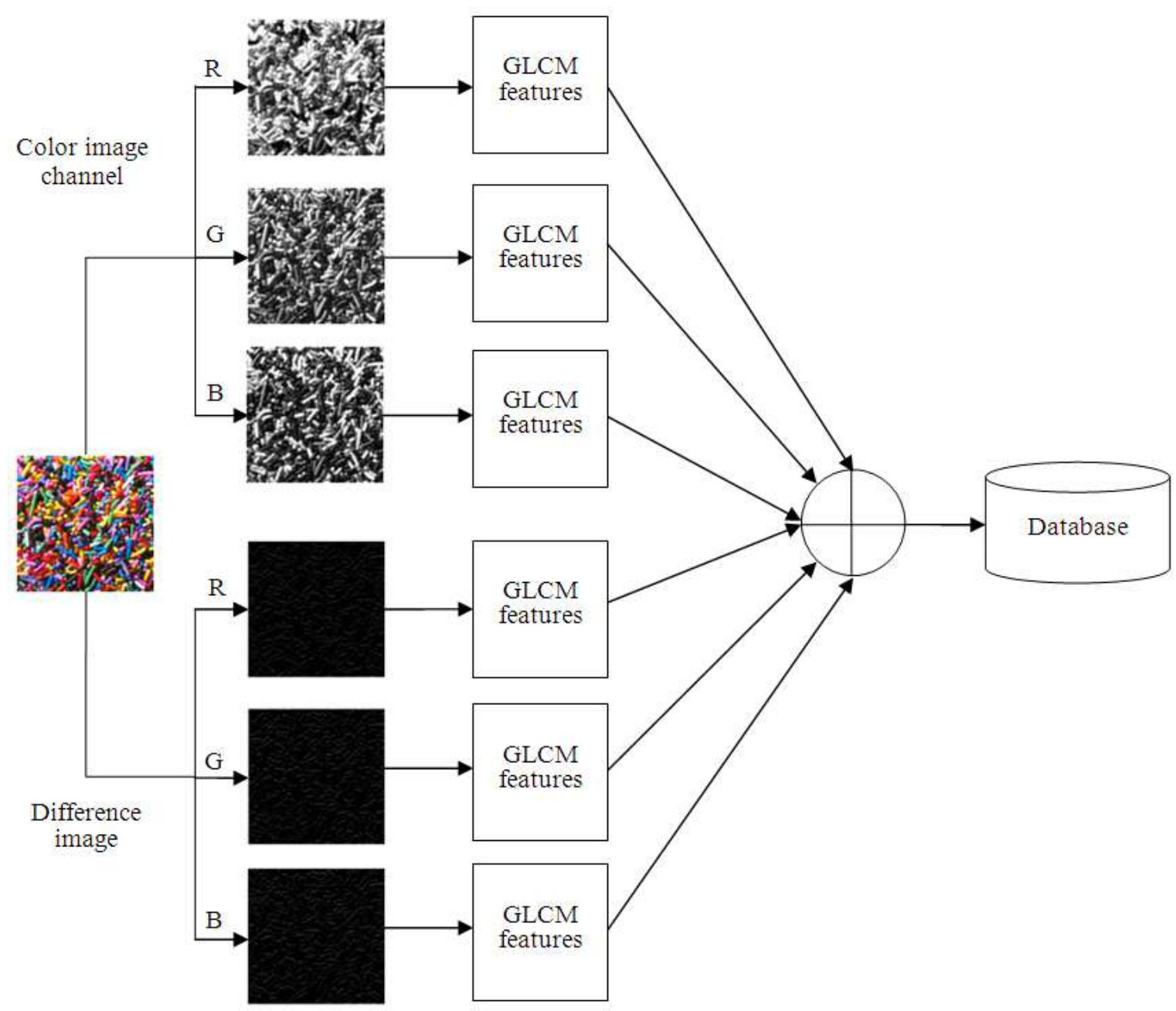

Fig. 2. Block diagram of the proposed colour texture classification system

Homogenity $=\sum_{i, j} \frac{p(i, j)}{1+|i-j|}$

\subsection{K-Nearest Neighbor Classifier}

In pattern recognition, the K-Nearest Neighbor algorithm $(\mathrm{K}-\mathrm{NN})$ is a method for classifying objects based on closest training examples in the feature space. $\mathrm{K}-\mathrm{NN}$ is a type of instance-based learning where the function is only approximated locally and all computation is deferred until classification. In K-NN, an object is classified by a majority vote of its neighbors, with the object being assigned to the class most common amongst its $\mathrm{k}$ nearest neighbors ( $\mathrm{k}$ is a positive integer, typically small). If $\mathrm{k}=1$, then the object is simply assigned to the class of its nearest neighbor. The neighbors are taken from a set of objects for which the correct classification is known. This can be thought of as the training set for the algorithm, though no explicit training step is required.

\subsection{Proposed Method}

The block diagram of feature extraction stage of the propoed colour texture classification system is shown in Fig. 2. In the proposed approach, the GLCM matrix is extracted for the difference image calculated along the first non singleton dimension of the input texture image. Let us consider a vector $\mathrm{X}$. The first non singleton dimension $\left(X^{\prime}\right)$ of the vector $X$ is one element shorter than $\mathrm{X}$, of differences between adjacent elements is given by system in Equation 5: 


$$
X^{\prime}=[X(2)-X(1) X(3)-X(2) X(n)-X(n-1)]
$$

From the difference vector or difference image, GLCM matrix is calculated and the statistical features are extracted. The same procedure is applied to all training samples samples and stored in the database. Finally, in the classification stage, the feature vector of the unknown texture image is extracted and processed with the features in the database generated in the feature extraction. Textures were classified using a KNN classifier by using the Euclidean distance measure. The performance of the proposed system is measured as the percentage of test set images classified into the correct texture class.

20 colour textures, obtained from the VisTex album is used in the experiments are shown in Fig. 3. Every original image is of size $512 \times 512$ pixels. 144 sample images of size $128 \times 128$ with an overlap of 32 pixels between vertically and horizontally adjacent images are extracted from each original image. Among the 144 images, 61 images are randomly selected for each colour texture images. 30 images are used in the training phase and the remaining 31 images are used for testing phase.

\section{RESULTS AND DISCUSSION}

The performance of the proposed system is carried out with each colour planes and the fusion of colour plane features. Table 1 shows the comparison of colour texture classification rate with different colour channels. R, G and B denote GLCM features extracted from the original Red, Green and Blue channels respectively. $\mathrm{R}^{*}, \mathrm{G}^{*}$ and $\mathrm{B}^{*}$ denote GLCM features extracted from Red, Green and Blue channels of difference image respectively. RGB and $R^{*} G^{*} B *$ denote the fusion of features extracted from the original and difference image.

The performance of the proposed system is compared with Hassan method in (Maia et al., 2010). They constructed hybrid colour texture space by using the most discriminating attributes of the textures. The Attributes used are calculated from the co-occurrence matrix. The minimum-Redundancy-MaximumRelevance feature algorithm is used for selecting the discriminating features. Support vector machine classifier is used and in comparision with an iterative selection is also made.

The same texture images used by Hassan are taken for evaluation. The selected 16 VisTex images are divided into 64 non-overlapping of $64 \times 64$ sized subimages. The first 30 sub-images of each texture are used for training the classifier and the remaining images are used for testing. The classification accuracy of the proposed system with Hassan method is given in Table 2.

Table 1. Classification rate of the proposed classification system based on GLCM

\begin{tabular}{|c|c|c|c|c|c|c|c|c|c|}
\hline Texture ID & $\mathrm{R}$ & $\mathrm{G}$ & $\mathrm{B}$ & $\mathrm{R}^{*}$ & $\mathrm{G}^{*}$ & $\mathrm{~B}^{*}$ & RGB & $\mathrm{R}^{*} \mathrm{G}^{*} \mathrm{~B}^{*}$ & $\mathrm{RR} * \mathrm{GG} * \mathrm{BB} *$ \\
\hline Bark.0006 & 77.08 & 84.72 & 97.22 & 76.39 & 84.03 & 83.33 & 95.14 & 91.67 & 97.92 \\
\hline Bark.0012 & 75.00 & 78.47 & 67.36 & 95.14 & 94.44 & 95.83 & 77.08 & 95.83 & 96.53 \\
\hline Brick.0000 & 92.36 & 99.31 & 96.53 & 79.86 & 77.08 & 68.75 & 100.00 & 89.58 & 98.61 \\
\hline Fabric. 0002 & 88.89 & 94.44 & 100.00 & 97.92 & 98.61 & 97.22 & 100.00 & 98.61 & 100.00 \\
\hline Fabric.0013 & 100.00 & 100.00 & 100.00 & 98.61 & 95.14 & 99.31 & 100.00 & 100.00 & 100.00 \\
\hline Fabric.0017 & 84.03 & 84.72 & 75.69 & 100.00 & 100.00 & 100.00 & 84.72 & 100.00 & 100.00 \\
\hline Flowers.0006 & 89.58 & 93.75 & 98.61 & 71.53 & 61.81 & 72.92 & 97.22 & 91.67 & 98.61 \\
\hline Food.0000 & 94.44 & 100.00 & 100.00 & 100.00 & 100.00 & 100.00 & 100.00 & 100.00 & 100.00 \\
\hline Food.0001 & 100.00 & 100.00 & 100.00 & 100.00 & 100.00 & 100.00 & 100.00 & 100.00 & 100.00 \\
\hline Food.0009 & 94.44 & 98.61 & 100.00 & 99.31 & 99.31 & 100.00 & 99.31 & 99.31 & 100.00 \\
\hline Grass.0001 & 96.53 & 96.53 & 97.92 & 100.00 & 100.00 & 100.00 & 96.53 & 100.00 & 99.31 \\
\hline Leaves.0012 & 95.14 & 90.97 & 80.56 & 100.00 & 100.00 & 100.00 & 99.31 & 100.00 & 100.00 \\
\hline Metal.0002 & 100.00 & 100.00 & 100.00 & 100.00 & 100.00 & 100.00 & 100.00 & 100.00 & 100.00 \\
\hline Metal.0004 & 95.14 & 99.31 & 99.31 & 100.00 & 100.00 & 100.00 & 98.61 & 100.00 & 100.00 \\
\hline Misc. 0001 & 100.00 & 100.00 & 100.00 & 95.14 & 89.58 & 97.22 & 100.00 & 100.00 & 100.00 \\
\hline Misc. 0002 & 100.00 & 100.00 & 100.00 & 97.22 & 97.22 & 96.53 & 100.00 & 99.31 & 100.00 \\
\hline Paintings.1.0001 & 91.67 & 94.44 & 94.44 & 84.03 & 74.31 & 93.75 & 96.53 & 93.75 & 100.00 \\
\hline Sand.0000 & 100.00 & 100.00 & 100.00 & 100.00 & 100.00 & 100.00 & 100.00 & 100.00 & 100.00 \\
\hline Sand.0002 & 100.00 & 100.00 & 100.00 & 92.36 & 97.22 & 100.00 & 100.00 & 100.00 & 100.00 \\
\hline Wood.0001 & 93.06 & 95.14 & 92.36 & 79.86 & 89.58 & 88.19 & 95.14 & 91.67 & 96.53 \\
\hline Average & 93.37 & 95.52 & 95.00 & 93.37 & 92.92 & 94.65 & 96.98 & 97.57 & 99.38 \\
\hline
\end{tabular}



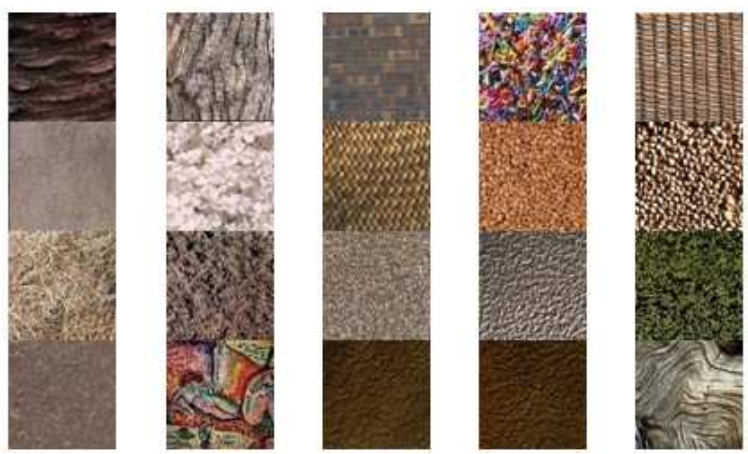

Fig. 3. VisTex colour texture images used in the experiments

Table 2. Classification accuracy of the proposed system with Hassam method in (Maia et al., 2010)

\begin{tabular}{|c|c|c|c|c|}
\hline Texture ID & $\begin{array}{l}\text { RGB } \\
\text { (Maia et al., } \\
\text { 2010) }\end{array}$ & $\begin{array}{l}\text { MIQ } \\
\text { (Maia et al., } \\
\text { 2010) }\end{array}$ & $\begin{array}{l}\text { Iterative } \\
\text { selection } \\
\text { (Maia } \text { et al., } \\
\text { 2010) }\end{array}$ & $\begin{array}{l}\text { Proposed } \\
\text { approach }\end{array}$ \\
\hline$\overline{\text { Bark12 }}$ & 60.7 & 63.0 & 65.0 & 100.00 \\
\hline Fabric0 & 98.9 & 99.6 & 99.2 & 94.12 \\
\hline Fabric8 & 61.6 & 64.4 & 66.2 & 61.76 \\
\hline Fabric11 & 77.7 & 86.3 & 85.8 & 100.00 \\
\hline Fabric17 & 73.9 & 82.4 & 80.8 & 55.88 \\
\hline Fabric19 & 83.5 & 85.0 & 84.1 & 44.12 \\
\hline Food0 & 97.2 & 98.2 & 98.6 & 97.06 \\
\hline Leaves 12 & 61.0 & 59.7 & 20.8 & 97.06 \\
\hline Grass1 & 55.8 & 65.5 & 64.4 & 100.00 \\
\hline Clouds0 & 99.5 & 98.2 & 97.8 & 82.35 \\
\hline Brick0 & 98.4 & 98.2 & 97.9 & 100.00 \\
\hline Wood0 & 91.2 & 90.7 & 90.5 & 88.24 \\
\hline Water0 & 59.5 & 56.5 & 49.9 & 88.24 \\
\hline Stone4 & 80.1 & 84.0 & 86.5 & 94.12 \\
\hline Sand0 & 99.0 & 99.0 & 99.0 & 44.12 \\
\hline Metal & 89.4 & 92.7 & 93.6 & 100.00 \\
\hline Average & 80.4 & 82.7 & 80.0 & 84.19 \\
\hline
\end{tabular}

Among the 16 texture image, 100\% classification accuracy is achieved by the proposed system. However, for Fabric19 and Sand0 texture images less than 50\% accuracy is obtained due to its random texture properties.

\section{CONCLUSION}

In this study, an efficient approach for the classification of colour texture images based on GLCM is proposed. The GLCM features, contrast, correlation, energy and homogeneity are extracted from each colour plane of the original image and the first non singleton difference image. These features are fused at feature level to classify the colour texture images. 20 VisTex album colour images are used to evaluate the proposed system. The classification accuracies obtained by the proposed feature fusion approach using nearest neighbor classifier are tabulated. From the experimental results, the proposed fusion of difference image GLCM feature provides better classification rate than the original image GLCM features.

\section{REFERENCES}

Akhloufi, M.A., X. Maldague and W.B. Larbi, 2008. A new color-texture approach for industrial products inspection. J. Multimedia, 3: 44-50. DOI: 10.4304/jmm.3.3.44-50

Bouguila, N. and R.I. Hammoud, 2009. Color texture classification by a discrete statistical model and feature selection. Concordia University, Montreal.

Bunte, K., I. Giotis, N. Petkov and M. Biehl, 2011. Adaptive matrices for color texture classification. Proceedings of the 14th International Conference on Computer Analysis of Images and Patterns, (AIP' 11), Springer-Verlag Berlin, Heidelberg, pp: 489-497.

Ershad, S.F., 2011. Color texture classification approach based on combination of primitive pattern units and statistical features. Int. J. Multimedia Appli., 3: 3-3. DOI: 10.5121/ijma.2011.3301

Hernandez, O.J., J. Cook, M. Griffin, C.D. Rama and M. McGovern, 2005. Classification of color textures with random field models and neural networks, J. Comput. Sci. Technol.

Maia, H.E., A. Hammouch and D. Aboutajdine, 2010. A color-texture approach based on mutual information for multispectral image classification. J. Multimedia, 5: 481-487. DOI: $10.4304 / \mathrm{jmm} \cdot 5.5 .481-487$

Palm, C. and T.M. Lehmann, 2002. Classification of color textures by gabor filtering. Mach. Graph. Visi., 11: 195-219.

Patil, N.K., V.S. Malemath and R.M. Yadahalli, 2011. Color and texture based identification and classification of food grains using different color models and haralick features. Int. J. Comput. Sci. Eng.

Sadat, R.M.N., S.W. Teng, G. Lu and S.F. Hasan, 2011. Texture classification using multimodal invariant local binary pattern. Proceedings of the IEEE Workshop on Applications of Computer Vision, Jan. 5-7, IEEE Xplore Press, Kona, HI., pp: 315-320. DOI: 10.1109/WACV.2011.5711520

Sengur, A., 2009. Color texture classification using wavelet transform and neural network ensembles. Arabian J. Sci. Eng., 34: 491-502. 
Sertel, O., J. Kong, G. Lozanski, A. Shana'ah and U.C.J. Saltz et al., 2008.Texture classification using nonlinear color quantization: Application to histopathological image analysis. Proceedings of the IEEE International Conference on Acoustics, Speech and Signal Processing, Mar. 31-4, IEEE Xplore Press, Las Vegas, NV., pp: 597-600. DOI: 10.1109/ICASSP.2008.4517680
Tou, J.Y., Y.H. Tay and P.Y. Lau, 2009. Recent trends in texture classification: A review. Pennsylvania State University.

Wouwer, V., G. Scheunders, P.S. Livens and D.V. Dyck, 1999. Wavelet correlation signatures for color texture characterization. Patt. Recogn., 32: 443-451. DOI: 10.1016/S0031-3203(98)00035-1 